\title{
Complex Assessment of Combat Neurotrauma
}

\author{
Bowen $\mathrm{M}^{* 1,3}$, and Chang $\mathrm{A}^{2}$
}

${ }^{1}$ Department of Military Science, James Madison University, Harrisonburg, USA

${ }^{2}$ Program in Cognitive Neuroscience, University of Virginia, Charlottesville, USA

${ }^{3}$ Independent Practice of Clinical Neuropsychology, Charlottesville, USA

*Corresponding author: Bowen M, Department of Military Science, James Madison University, Harrisonburg, VA, 22807, USA, Tel: 434-962-4720, E-mail: drmbowen@comcast.net

Citation: Bowen M, Chang A (2018) Complex Assessment of Combat Neurotrauma. J Neurol Neurol Disord 4(2): 107

Received Date: March 11, 2018 Accepted Date: June 22, 2018 Published Date: June 25, 2018

\begin{abstract}
This study explores the patterns of twelve male OIF-OEF combat veterans with the Tactual Performance Test (TPT) in cases of Posttraumatic Stress Disorder (PTSD) and blast-related mild traumatic brain injury (mTBI) or with PTSD only. 6 were dual diagnosed with PTSD+mTBI and 6 with PTSD-o. Last deployment ranged from 10 to 3.5 years. Results suggest themes of lateralization, intertrial reversals of both regression and rebound, and discrepancies between memory and localization. Findings are discussed in relation to both the conceptual underpinnings and application of the TPT and the functional neuroanatomy of PTSD and blast-related mTBI.
\end{abstract}

Keywords: Tactual Performance Test; Blast-related mTBI; PTSD

\section{Introduction}

\section{The Tactual Performance Test}

The Tactual Performance Test (TPT) is one of the measures comprising the Halstead-Reitan Neuropsychological Test Battery (HRB). While developed to uniquely assess a blend of higher cortical functions [1,2] its comparative complexity of administration and exceptional potential to stress the patient have lent to comparatively uncommon use in clinical practice outside of strict application within the HRB [3,4].

Utilizing a form board with "cookie-cutter" spaces and wooden blocks to fill the spaces (adult version - 10; pediatric versions - 6), the subject is blindfolded and given no prior or subsequent view of the board. The standard procedure entails successive trials using the dominant, non-dominant and both hands. Six measures are obtained: 1) dominant hand time; 2) non-dominant hand time; 3) bimanual time; 4) total time; 5) number of shapes recalled, and 6) location of recalled shapes. The latter two measures are obtained upon removing the blindfold and asking the subject to attempt duplication of block shapes and their location using paper and pencil (this final procedure is untimed).

The TPT is a comparatively complex assessment instrument both for the above procedural demands and further its multi-modal analysis of function. The test's design and procedure assesses the two cerebral hemispheres for comparison and provides evidence of the general efficiency of brain functions. The TPT is a demanding task in terms of its motor and sensory requirements [5]. The procedure incorporates motor functioning, tactile form discrimination, problem solving, spatial reasoning, and memory skills. The mnestic factor demands both working through intermediate-term recall for tactile and spatial stimuli. Normals show an approximate 30\% improvement across the first two trials of the TPT, but with deviation of this tendency not uncommon in older subjects [6,7]. In a classic comprehensive review, it was concluded that the TPT appears to be as valid an indicator of brain impairment as any other single neuropsychological test, and furthermore that: 1) this is moreso for the timed scores than for Memory or Location; 2) with Location more sensitive than Memory; 3) while indeed detecting brain damage per se, it may be most discriminative for posterior and/or right hemisphere damage; 4) reliability of the TPT measures are adequate; 5) the emphasis on quantitative scores need not diminish qualitative aspects [8]. Reliability co-efficients were provided in a study drawing from large samples of normal subjects and neurologic patients. Findings were of Total Time to completion $r=.866$ in normals and $r=.873$ in the patient group [9]. 


\section{Functional Neuroanatomy of Posttraumatic Stress Disorder}

Posttraumatic stress disorder (PTSD) symptoms are considered related to an individual's dysregulated biological response to stress. Research has largely focused on the hypothalamus- pituitary-adrenal (HPA) axis, with parallel exploration of the sympatheticadrenomedullary (SAM) system and its implications for production of epinephrine facilitating the flight or fight response [10]. In their functional imaging meta-analysis of symptom provocation in PTSD, there were findings of significant negative co-activation between frontal cortical versus limbic and paralimbic regions, wherein frontal cortical regions were hypoactive and limbic/ paralimbic hyperactive [11]. Affected cortical regions were medial pre-frontal (mPFC) with involved subcortical regions being the anterior cingulate cortex (ACC), amygdala and insula. Compared to other anxiety syndromes, the limbic hyperactivity associated with decreased frontal inhibition was a unique feature of PTSD. Further unique to PTSD was significant hypoactivation of the thalamus, postulated to relate to decreased processing of sensory information.

In a comprehensive review of structural, functional, and receptor alterations associated with PTSD, Garfinkel \& Liberzon (2009) reported similar results converging on the amygdala, ACC, mPFC, insula, and additionally the hippocampus. Structurally the predominant findings were of reduced volumes and functionally with activation patterns reflecting those found by Etkin $\&$ Wager $[11,12]$. Of further note is that the neuroanatomical relationships with combat veterans are similar to those of other PTSD subgroups.

\section{Blast-related Brain Injury}

Nearly two million troops have been deployed to Operations Iraqi and Enduring Freedom (OIF-OEF). Traumatic brain injury secondary to an improvised explosive device (IED) has been characterized as the "signature injury" of the war, with explosive blasts accounting for the majority of combat casualties [13,14]. A comprehensive survey of returning veterans revealed over $80 \%$ of cases reporting combat duty loss of consciousness were due to a blast [15]. While the incidence of blast-related brain injuries has been estimated to be as high as over 300,000, a more recent review asserts that a reliable statistic cannot be asserted due to a multitude of factors [16,17]. Moreover, mTBI may have been over-diagnosed by late retrospective review of returned servicemen and women using imprecise criteria (Rosenfeld \& Ford, 2010).

Understanding of how a blast affects the brain is gaining from the recent growth of studies. Blast over-pressure both intracranial and simultaneously to air-filled organs is a very different traumatic process than acceleration-deceleration or blunt force induced brain damage. Theoretical understandings and clinical consequences are complicated by the secondary, tertiary and quaternary factors of: penetrating or striking debris and projectiles; the victim being forcefully propelled and making impact; and finally toxic inhalations, burns, and impact or entrapment from adjacent collapsing structures or vehicles [18]. Cognitive assessment of blastinduced mTBI is greatly confounded by the very high rates of co-occurring posttraumatic stress disorder and that syndrome's functional presentation and neurobiology (Stein \& McAllister, 2009) [12,19]

Sophisticated neuroimaging is allowing exquisite insight into the precise anatomy of blast mTBI Peskind et al. (2010) conducted the first fluorodeoxyglucose positron emission tomography (FDG-PET) study comparing blast-injured service members to controls and found decreased cerebral metabolic rates in the cerebellum, vermis, pons and medial temporal lobes in that order [20]. Magnetic resonance diffusion tensor imaging (DTI) has been applied to the population in very recent years. Warden $e t$ al. (2009) described a single soldier with blast-exposure post-concussion syndrome associated with isolated left cerebellar axonal damage on DTI [21]. In a sample of 63 service members symptomatic for mTBI after blast-exposure, in order of significance abnormalities were revealed in the cerebellar peduncles, cingulum bundles and right orbitofrontal white matter [16]. It was noted, however, that none of the subjects had isolated primary blast injury. In a subsequent small study of 4 patients with blast-related 'mild' traumatic brain injury isolated from any other known insult, DTI abnormalities were consistent with cerebellar white matter injury in 3 of the subjects. No abnormalities in other brain regions were detected. These findings added to the evidence supporting the hypothesis that primary last exposure contributes to brain injury in the absence of head impact and that the cerebellum may be particularly vulnerable [16]. Jorge et al. (2012) conducted DTI with 72 service members with blast-related mTBI, finding evidence of multi-focal white matter pathology associated with severity of injury [22]. Particularly for the more severely injured sub-group, damage was most apparent in the cerebellum, corpus callosum, and at the pontine-medullary juncture adjacent the fourth ventricle. The study also compared a smaller group of civilians with mTBI and found them to have greater evidence of DTIdetected pathology. Finally within the scope of this limited review, Adam et al. (2013) analyzed 106 blast-injured service members with DTI within 7 days of injury in the first use of MRI in a combat zone [16]. Findings were of significantly reduced white matter density in mTBI patients, most prominently the right superior longitudinal fasciculus, followed by the cerebellar middle peduncle and bilateral orbitofrontal zones. The results were viewed as reflecting the previous study (MacDonald et al. NEJM 2011) while also offering new evidence that white matter injury can occur even in milder forms of blast-related mTBI [16]. Finally, Graner et al. (2013) conducted a comprehensive review of imaging of blast-related mTBI and concluded that while little is known about the precise neurobiological effects of primary blast-waves on the brain, particularly in milder cases current clinical imaging methods cannot adequately diagnose the injury and both task-based and resting state fMRI have the potential to provide useful objective diagnostic information associated with functional sequelae [23]. 


\section{Comparative and Neurocognitive Studies of Blast-related mTBI and PTSD in Combat Veterans}

Schneiderman, Braver and Kang (2008) undertook a large survey to attempt to ascertain histories of combat theater injury mechanisms and mild traumatic brain injury (mTBI) and current prevalence of posttraumatic stress disorder (PTSD) and postconcussive symptoms [24]. The overall core finding was that the strongest factor associated with post-concussive symptoms was PTSD. Benge et al. (2009) found PTSD shared a statistically significant amount of variance with every post-concussion symptom across 345 veterans, and emphasized this finding being consistent with civilian data, albeit with the notion that somatic symptomsprimarily vestibular, headache and multi-sensory-might fractionate in a way that is unique to a veteran polytrauma sample [25]. To analyze the degree of PTSD symptoms in blast v. non-blast-related mTBI, Kennedy et al. (2010) compared samples of veterans and reported higher rates of flashbacks and nightmares in the blast injured group but without group differences either in the remaining range of symptoms nor in the total score [26]. Examining the symptom co-occurrence spectrum in a very large combat-exposed sample, Macera et al. (2012) compared mTBI and PTSD symptom reporting immediately and several months after deployment [27]. Their findings were of subjects reporting either condition alone to be inclined to also report the symptom cluster of the other condition later, and/or for those reporting either or both condition at onset to endorse worsening symptoms later. Greene et al. (2014) applied multimodal MRI to a small sample of post-9/11 deployed veterans, 13 with PTSD and blast exposure and 7 with neither. No significant differences between groups were found in medial prefrontal or parietal gray matter volume [28]. Most recently Garber, Rusu and Zamorski (2014), analyzed results of screening with a large post-deployed Canadian sample and concluded that mTBI had no significant association with post-concussive symptoms (PCS) relative to non-mTBI injury, but that in contrast, mental health problems per se had a strong association with the reporting of 3 or more PCS [29]. Most recently Hayes et al. (2015) found heterogeneous white matter abnormalities in OIF/OEF veterans with blast-related mTBI associated with loss of consciousness (LOC) [30]. Moreover, the mTBI/LOC group showed reduced verbal memory. PTSD was not associated with white matter abnormalities.

\section{Neurocognitive Studies}

Although to date the literature does not support blast-related brain injury as yielding a unique profile, there is controversy as to whether neurocognitive examination can distinguish it from the factors of PTSD and associated emotional syndromes. Crowell et al. (2002) assessed cognitive function across groups of active $\mathrm{v}$ [31]. inactive PTSD v. non-PTSD psychiatric v. controls. In that groups did not differ on the neuropsychological measures and veterans with PTSD performed similarly to demographically matched controls, the authors interpreted the results as suggesting that the cognitive difficulties previously linked to PTSD may actually have been secondary to pre-existing individual differences or other clinical conditions coexisting with PTSD. Fear et al. (2008) presented data from UK veterans that indicated a strong relationship between PCS (post-concussion syndrome) symptoms and PTSD [32]. Their finding was supported by PCS symptoms being associated with factors having no basis in mTBI.

Belanger et al. (2009) compared the neuropsychological results of patients with mild or moderate/severe mTBI acquired from non-blast trauma [18]. Overall, they found no differences as a function of mTBI etiology. Effects of injury severity were primarily a factor of PTSD symptoms. The study determined that cognitive impairment was determined more by severity than mechanism of injury on the verbal learning and memory measures administered. In a sample of 53 veterans with a history of mTBI with and without co-morbid PTSD, Nelson et al. (2010) found the co-morbid group scored significantly worse on measures of processing speed and executive functioning [33]. In a study by Brenner et al. (2010), 45 soldiers reporting blast-related mTBI were examined [34]. The sub-group with persisting symptoms of mTBI did not distinguish on neurocognitive measures from those reporting resolution. Moreover, test data on those reporting persistent PTSD did not differ from those without the diagnosis. Luethcke et al. (2011) compared concussive and psychological symptoms and cognitive performance between blast versus non-blast induced mTBI [35]. Their results suggested unconvincing distinction in concussive or psychological symptoms and neurocognitive performance between blast and non-blast mTBI, although clinically significant impairment in cognitive reaction time for both blast and non-blast groups was found. Cognitive impairment was related to duration of loss of consciousness rather than injury mechanism. Belanger et al. (2011) found a blast mechanism of brain injury does not differ from a non-blast mechanism of injury in terms of post-concussion symptom complaints in those with a reported history of mTBI [36]. Those reporting higher levels of PTSD and longer duration since injury were both independently associated with higher levels of PCS reporting. The only symptom that significantly varied between groups was hearing, wherein the blast-injured group reported more severe difficulty. Aupperle and colleagues (2012) summarized investigations regarding executive function and PTSD, and identified subtle impairments in response inhibition and attention regulation among those with PTSD [37]. Lange et al. (2012) compared blast with non-blast mTBI in service members and found a medium effect for depression and stress greater within the blast-related group, but after controlling for this found no differences in the neurocognitive data [38]. Nelson et al. (2012) evaluated neurocognitive outcomes in veterans at least 1.5 years returned from deployment with blast-related mTBI, Axis I psychopathology (1/3 PTSD), co-morbid diagnosis or combat-controls [39]. The conclusion was that remote mTBI does not contribute in isolation to cognitive impairment but rather that when present deficits are subtle and most likely attributable to PTSD and other purely psychological syndromes. In a large sample of 760 US Army soldiers uniquely assessed pre- and post-deployment, Vasterling et al. (2012), concluded that mTBI has limited lasting neuropsychological consequences while PTSD and depression are associated with more enduring cognitive compromise [40]. Shandera et al. (2013) examined cognitive performance in groups of mTBI+PTSD, PTSD-o, mTBI-o 
and Combat Controls [41]. They reported poorer performance in the mTBI+PTSD and PTSD-o groups, while mTBI-o did not perform significantly different from the CC group on any neurocognitive tests. Most recently Bolzenius et al. (2015) describe objective cognitive and self-reported findings in veterans with blast-related mTBI compared to civilian mTBI independent of co-morbid PTSD symptoms [42]. Their analyses failed to reveal differences on any individual cognitive test, but with veterans reporting more psychological and somatic complaints. Thus in the post-acute phase, subjective complaints related to blast-related mTBI do not co-vary with objective cognitive performance and cognitive outcomes from blast-related mTBI were similar to those of civilian forms of mTBI.

These and other studies; Karr, et al. 2014) of neuropsychological function in combat veterans-primarily of the Mideast Wars suggest an emphasis on measures of executive function, speed of information processing, verbal fluency and to a lesser extent visuospatial abilities [43-45]. More recently Troyanska et al. (2015) compared groups of OIF/OEF/OND veterans for the effect of blast-related mTBI, PTSD symptoms and combat exposure on cognitive performance [46]. While a history of mTBI was associated with greater endorsement PTSD and general combat exposure symptoms, there were no group differences in performance on cognitive measures.

We find no studies wherein the TPT has been administered to the combat veteran population. Indeed, already bearing in mind the aforementioned remarks contraindicating its use with civilians, it becomes quite understandable that clinical research has avoided subjecting traumatized veterans to prolonged blindfolding during a challenging, novel task. However, the pervasive neuroanatomy captured by the TPT's exceptional scope of simultaneous motor-sensory and higher cortical functions may well reflect those regions and connecting networks now shown to be vulnerable to PTSD and blast-related mTBI.

\section{Method}

\section{Participants}

Twelve male combat veterans ranging in age from 24-40 and education twelve to sixteen years were administered the TPT either strictly as research volunteers secondary to their participation in the Combat Veterans Oral History Project ( $\mathrm{n}=7)$, or as part of a clinical evaluation on referral from a veteran's organization $(n=5)$. Inclusion criteria required a Department of Defense certified diagnosis of PTSD and/or blast-related mTBI incurred in combat operations in Iraq or Afghanistan. All subjects were assessed as putting forth a best effort with no distinctions between test taking conditions. Clinically tested subjects were furthermore unremarkable on a sensory-motor measure demonstrated to illuminate symptom magnification [47]. For consistency of test exposure, the clinical evaluation subjects were first administered the TPT. 6 subjects were dual diagnosed with PTSD+mTBI, 6 with PTSD-o (only). All diagnoses pre-dated participation. Last deployment ranged from 10 to 3.5 years.

\section{Procedure}

All subjects were examined by the senior author, a practicing board-eligible clinical neuropsychologist with thirty years experience. Beyond the standard summary time per trial, time to placement block-by-block was recorded for all participants. Moreover, order of placement block-by-block was recorded for nine participants (eight research one clinical). Summary scores were calculated by the demographically-weighted manual of Heaton, Grant \& Matthews (1991) [48].

\section{Data Analysis}

\begin{tabular}{|c|c|c|c|c|c|c|c|c|}
\hline Diagnoses & $\begin{array}{l}\text { Dominant Hand } \\
(\mathrm{DH})^{1}\end{array}$ & $\begin{array}{l}\text { Non-dominant } \\
\text { Hand }(\mathrm{NDH})^{1}\end{array}$ & Both Hands $(\mathrm{BH})^{1}$ & $\begin{array}{c}\text { Total } \\
\text { Time }^{1}\end{array}$ & Memory $^{1}$ & $\begin{array}{l}\text { Locali- } \\
\text { zation }\end{array}$ & Mean & SD \\
\hline \multicolumn{9}{|c|}{ PTSD+mTBI } \\
\hline 1 & 4 & 17 & 2 & 8 & -8 & -2 & 3.50 & 8.57 \\
\hline 2 & 12 & -5 & -5 & 2 & 8 & -3 & 1.50 & 7.18 \\
\hline 3 & -5 & -8 & -12 & -7 & 7 & -12 & -6.17 & 7.03 \\
\hline 4 & 8 & -6 & -4 & 0 & -16 & 5 & -2.17 & 8.59 \\
\hline 5 & -15 & -7 & -11 & -12 & -3 & -2 & -8.33 & 5.20 \\
\hline 6 & -2 & -6 & 12 & -1 & -12 & -10 & -3.17 & 8.59 \\
\hline \multicolumn{9}{|c|}{ PTSD-o+Be ${ }^{2}$} \\
\hline 7 & -7 & -5 & -18 & -14 & -10 & -10 & -10.67 & 4.72 \\
\hline 8 & -7 & 0 & -12 & -8 & -1 & -6 & -5.67 & 4.50 \\
\hline 9 & -7 & 3 & -9 & -6 & 8 & 1 & -1.67 & 6.68 \\
\hline 10 & 0 & -1 & -5 & -2 & 18 & -3 & 1.17 & 8.42 \\
\hline 11 & 4 & 0 & -4 & -1 & 8 & 24 & 4.00 & 10.13 \\
\hline 12 & -3 & -17 & -16 & -13 & 8 & -10 & -8.50 & 9.52 \\
\hline
\end{tabular}


Participant data were collected as time (minutes and seconds) for the timed trials, and number of correct responses in the Memory and Localization tasks. These data values were then converted to a T-Score using normative conversion tables, wherein T-Score conversions are based on specific participant demographics [48]. The means and standard deviations for each participant were calculated from the T-Scores. Participants' diagnoses, T-Scores, means, and standard deviations can be found in Table 1. Using the standard deviations calculated from the T-Scores, a one-tailed Comparison of Means t-Test was performed in order to assess the variance in TPT results within this population as compared to the variance in TPT results within the normal population.

\section{Results}

Results of the Comparison of Means t-Test can be found in Table 2. Assuming the mean standard deviation of the normal population is less than $6.37\left(\mathrm{M}_{\mathrm{SD} \text { of population }}=5.5\right.$ in Comparison of Means t-test) there are significant t-test results showing that on average, the present sample is more variant in their TPT results than members of the normal population. Although $\mathrm{M}_{\mathrm{SD} \text { of population }}$ was chosen arbitrarily, the comparison of means t-test is robust to conservative arbitrary values due to a small confidence interval: we are $95 \%$ confident that if we took more measurements from the affected population, their means would lie between 6.37 and 8.48 .

\begin{tabular}{|c|c|}
\hline Count & $\mathbf{1 2}$ \\
\hline Sample Mean & 7.43 \\
\hline 95\% Confidence Interval & $6.37-8.48$ \\
\hline SD & 1.87 \\
\hline SE & 0.54 \\
\hline Hypothesized Population Mean & 5.5 \\
\hline Alpha & 0.05 \\
\hline Tails 1 & 1 \\
\hline df & 11 \\
\hline t-statistic & 3.58 \\
\hline p-value & 0.002 \\
\hline t-critical & 2.20 \\
\hline
\end{tabular}

${ }^{1} \mathrm{~A}$ two-tailed t-test was not considered because it is unreasonable to believe that the variations can be both significantly high and significantly low. However, results are still significant when conducting a two-tailed t-test, $\mathrm{p}=0.004$.

Table 2: Comparison of Means t-Test using Standard Deviations within Participant T-Scores

Findings depicting abnormal inter-trial patterns are shown in Table 3. In the normative data across the four very similar demographic equations represented by participants, at $\mathrm{T}$ score 50 the midpoint time difference between consecutive trials is: $1>2=24 \%$ and $2>3=42 \%$. The present data yield regressions between trials in 5 of 12 participants with $3 / 5$ of those between trials 1 and 2.4 of the 7 remaining participants show exceptional non-regressive differences between trials wherein $3 / 4$ are due to lack of versus exceptional improvement and likewise 3/4 due to the differences occurring between trials 2 and 3. Overall these data reveal that $11 / 12(91.6 \%)$ of subjects had an inter-trial pattern deviating from the norm. Whether or not a subject had a Total Time score in the average range was not a factor in the stability of the inter-trial pattern wherein 9/12 (75\%) of subjects were within $1 \mathrm{~T}$ score of 50 , with near equal distribution greater $(\mathrm{N}=4)$ or less than $(\mathrm{N}=5)$. The findings are nearly equal between diagnostic groups but with the PTSD-o group yielding a trend toward greater non-regressive abnormality between trials.

\begin{tabular}{|c|c|c|c|}
\hline Subject & $\begin{array}{c}\text { Regressions } \\
\text { Between Trials }\end{array}$ & $\begin{array}{c}\text { Other Abnormal } \\
\text { Intertrial Data }\end{array}$ & $\begin{array}{c}\text { Total Time } \\
\text { TScore }\end{array}$ \\
\hline 1 & $3>2$ by $\%$ & & 58 \\
\hline 2 & $2>1$ by $13 \%$ & & 52 \\
\hline 3 & & & 43 \\
\hline 4 & $2>1$ by $21 \%$ & & 38 \\
\hline 5 & & $3<2$ by $66 \%$ & 49 \\
\hline 6 & & & 36 \\
\hline 7 & $3>2$ by $12 \%$ & $3<2$ by $4 \%$ & 41 \\
\hline 8 & & & 44 \\
\hline 9 & & $2<1$ by $10 \%$ & 49 \\
\hline 10 & & & 53 \\
\hline 11 & & $2>1$ by $53 \%$ & 38 \\
\hline 12 & & & 38 \\
\hline
\end{tabular}

Table 3: Abnormal Intertrial Patterns within Participants 
Further understandings both quantitative and qualitative are offered by content analysis of the regression trial participants as block placement times within trials shown in Table 4. These data suggest several trends: one or two extraordinarily long block placements within a trial most frequently account for the overall poor result versus a slow performance throughout a trial; significant slowing in a block or across half a trial always occurred in the second half of a trial and as isolated blocks most frequently as the last block; scores for memory and location-and to a lesser extent location-do not suggest a contributing factor in these data.

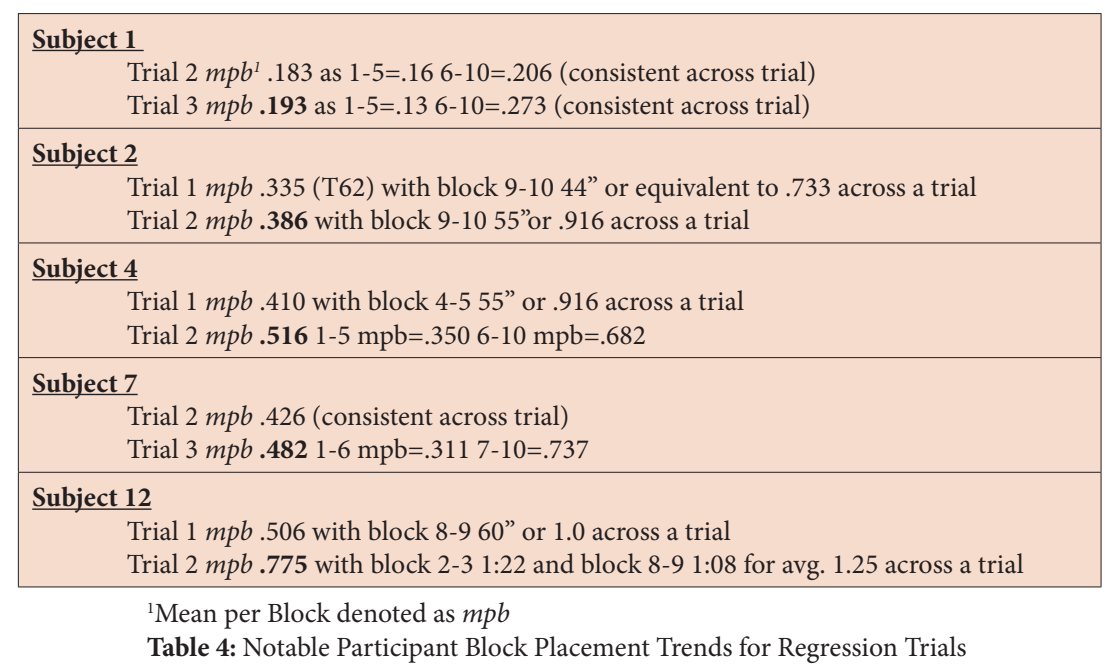

Among the nine participants tracked for placement order of each block, there were only two instances of the first block placed in a trial being again placed first in the subsequent trial. There was not a single instance of the last block in a trial being placed as the first block in the subsequent trial. Overall content analysis yields no evidence of any consistency of serial block placements between trials, and with higher overall result scorers for either total time or memory/location as random in block placement as low overall scorers.

\section{Discussion}

This first study to our knowledge applying the Tactual Performance Test to a sample of combat traumatized young males portrays its value in detecting related impairment in higher cortical function. A diagnosis of blast-related mTBI and PTSD versus PTSD-o did not discriminate either quantitative or qualitative performance patterns.

Thompson et al. (1987) asserted that "truly consistent, deviant performances are quite rare in normal subjects." The current abnormal findings between Trials 1 and 2 reflect the earlier study (Thompson et al. 1987) in respect to much older or lesser educated subjects [49]. The present data further portray that the inter-trial deviations from normal similarly occurred between Trials 2 and 3 .

While the present sample is overwhelmingly more variant in their TPT results than the normal population, their exceptional fluctuations both between and within trials by no means reflects an impaired result for Total Time, Memory or Location. Only one-quarter of the sample scored greater than one standard deviation below normal for Total Time, and in each of those cases by a marginal degree. However, all but one of the dozen subjects yielded abnormal inter- or intra-trial findings. Thus the key finding of this study is of a qualitative / process nature.

Thompson \& Parsons (1985) urged that "the emphasis on quantitative results need not diminish the use of qualitative aspects as well." Indeed, the findings of this combat traumatized sample suggest a strong trend of waning and/or temporary derailing in the test taking process [8]. Thus the pattern for the sample was to diminish rather than accelerate in the second half of a trial, regardless of the trial. A reverse pattern is expected given that there is both lesser stimuli to cope with in the latter half of a trial and because learning should occur over trials given that the stimuli do not vary. The factor of derailing within trials refers to one or two blocks (again, in the latter half of a trial) consuming an inordinate amount of time within a trial.

The TPT's founding purpose and longer-term history as a tool for discriminating lateralization of dysfunction is obviated by the diffuse white matter and/or regionally bilateral and sub-cortical nature of impairment resulting from blast-related brain injury and PTSD. Because only half of the subjects in the current sample had a diagnosis of blast-related mTBI while all subjects had a diagnosis of PTSD, and, accumulating recent studies conclude that long-term cognitive deficits appear attributable to PTSD rather than mTBI, postulating the neurosubstrates of the present sample's impaired process on the TPT need necessarily focus on the PTSD factor. The Etkin and Weger (2007) functional imaging meta-analyses may be particularly heuristic, and in particular its finding that unique to PTSD was significant hypoactivation of the thalamus and resultant decrease of sensory processing [11]. 
In their classic treatise, Llinas et al. (1998) provide detailed evidence for thalamocortical resonance as the substrate of consciousness [50]. The underpinning of that assertion was their finding that the temporal interval for sensory discrimination is determined by synchronous activity in the thalamocortical system, and furthermore that the system is viewed as encoding specific sensory and motor activity and associative functions. They emphasize that the system functions on the basis of temporal coherence in synchronicity with distal and proximal activity. Quite reasonably, these phenomena suggest the cognitive neurodynamics evoked by the TPT.

Similarly worth considering is the review of Cavanna \& Trimble (2006) [51]. Focusing on the function of the precuneous-the posteromedial portion of the parietal lobe with dense thalamic connections-they illuminate its key role in a wide spectrum of highly integrated tasks including visual-spatial imagery, episodic memory retrieval and self-processing operations, all neurocognitive functions with a particularly strong load for executing the TPT. The authors emphasize that the precuneous, by way of subcortical and cortical networks, is centrally involved in the specialized process of spatially guided behavior. Their discussion asserts that the integrated elaboration of spatial relations and motor imagery, concurrent with higher-order processes of voluntary attentional shifts and more abstract mental imagery, all emanate from the precuneous. They further elaborate that the precuneous is central to directing attention in space during the execution of goal-directed movements and during tasks requiring spatial information about the direction of movements in an imaginary field. This function also occurs when a visuospatial stimulus is processed just mentally without the concurrent execution of motor activity. Because the precuneous activates at the onset of imagined movement, it is postulated to be important to the generation of spatial information, specifically as episodic memory retrieval and mental imagery strategies. The nature of the TPT quantitative and qualitative results with the current sample are very strongly indicative of impairment in those two functions. An fMRI study utilizing the TPT might contribute to the confirmation of these findings for the function of the precuneous.

While the current study provides insightful introductory understandings of the value of administering the TPT to younger veterans diagnosed with the two most common chronically disabling brain syndromes of Middle East War combat, its limitation is of course the small sample(s). Further studies utilizing the TPT with veterans diagnosed with either or both conditions are needed to crystallize and possibly expand upon the current findings.

We respectfully differ with the long ago assertion of Lezak (1983) that the TPT is not worth the time and strain incurred by the patient [4]. Moreover, it is the experienced opinion of the senior author that the test is perhaps a particularly good fit for the combat veteran, for they are exceptionally inclined and accustomed to an arduous manual task and seem to engage the gross-motor reliant TPT with an eagerness often not apparent in their undertaking of other tests commonly used for clinical and research purposes with their population. Thus the clinician is encouraged not to shy from use of the TPT in assessment of the combat veteran. An accumulation of TPT data with the combat trauma patient population will serve to enhance understandings of the value of the instrument within the broader evaluation process. Future research with the instrument is encouraged to specifically pursue intraand inter-trial quantitative and qualitative data with both normal and patient populations.

\section{References}

1. Halstead W (1947) Brain and intelligence. University of Chicago Press, Chicago.

2. Reitan RM, Davison LA (1974) Clinical neuropsychology: Current status and application. Wiley, New York.

3. Klove H, Reitan R, Davison L (1974) Current status of clinical neuropsychology. In: RM Reitan \& LA Davison (Edn) Clin Neuropsychol Cur Sta Appli 325-61.

4. Lezak MD (1983) Neuropsychological Assessment. ( $2^{\text {nd }}$ Ed) Oxford University Press, New York.

5. Reitan RM, Wolfson O (1993) The Halstead-Reitan Neuropsychological Battery: Theory and clinical interpretation (2 ${ }^{\text {nd }}$ Edn). Neuropsychology Press, AZ, USA.

6. Filskov SB, Boll TJ (1981) The Halstead-Reitan Neuropsychology Batter. In: Filskov SB, Boll TJ (Edn), Handbook of clinical neuropsychology, $577-607$.

7. Thompson LL, Heaton RK (1991) Pattern of Performance on the Tactual Performance Test. Clin Neuropsychol 5: $322-8$.

8. Thompson LL, Parsons OA (1985) Contribution of the TPT to Adult Neuropsychological Assessment: A Review. J Clin Exp Neuropsychol 74: 430-44.

9. Charter RA (2001) Tactual Performance Test Trials: Internal Consistency Reliability Using The Gilmer-Feldt Coefficient. Percept Mot Skills 93: 363-6.

10. Brenner LA (2011) Neuropsychological and Neuroimaging Findings in Traumatic Brain Injury and Post-traumatic Stress Disorder. Dialogues Clin Neurosci 13: 311-23.

11. Etkin A, Wager TD (2007) Functional Neuroimaging of Anxiety: A Meta-Analysis of Emotional Processing in PTSD, Social Anxiety Disorder, and Specific Phobia. Am J Psychiatry 164:1476-88.

12. Garfinkel SN, Liberzon I (2009) Neurobiology of PTSD: A Review of Neuroimaging Findings. Psychiatric Annals 39: $370-81$.

13. DePalma RG, Burris DG, Champion HR, Hodgson MJ (2005) Blast Injuries. N Eng J Med 352: 1335-42.

14. Ling G, Bandak F, Armonda R, Grant G, Ecklund J (2009) Explosive Blast Neurotrauma. J Neurotrauma.26: 815-25.

15. Hoge CW, McGurk D, Thomas JL, Cox AL, Engel CC, et al. (2008) Mild traumatic brain injury in U.S. soldiers returning from Iraq. N Engl J Med 358: 453-63. 16. Adam O, MacDonald C, Rivet D, Ritter J, May T, et al. (2013) Diffusion Tensor Imaging in Acute Blast-Related Mild Traumatic Brain Injury in Injured Service Members in Afghanistan. Neurol 80: S14.

17. Bagalman E (2013) Traumatic Brain Injury Among Veterans. CRS Report for Congress 7-5700.

18. Belanger HG, Kretzmer T, Yoash-Gantz R, Pickett T, Tupler LA (2009) Cognitive Sequelae of Blast-related Versus Other Mechanisms of Brain Trauma. J Int Neuropsychol Soc 15: 1-8. 
19. Polusny MA, Kehle SM, Nelson NW, Erbes CR, Arbisi PA, et al. (2011) Longitudinal effects of mild traumatic brain injury and posttraumatic stress disorder comorbidity on post-deployment outcomes in National Guard soldiers deployed to Iraq. Arch Gen Psychiatry 68: 79-89.

20. Peskind ER, Petrie EC, Cross DJ, Pagulayan K, McCraw K, et al. (2010) Cerebrocerebellar hypometabolism associated with repetitive blast exposure mild traumatic brain injury in 12 Iraq war veterans with persistent post-concussive symptoms. Neuroimage 54: S76-82.

21. Warden DL, French LM, Shupenko L, Fargus J, Riedy G, et al. (2009) Case Report of a Soldier with Primary Blast Brain Injury. NeuroImage 47: T152-3.

22. Jorge RE, Acion L, White T, Tordesillas-Gutierrez D, Pierson R, et al. (2012) White Matter Abnormalities in Veterans With Mild Traumatic Brain Injury. Am J Psychiatry 169: 1284-91.

23. Graner J, Oakes TR, French LM, Riedy G (2013) Functional MRI in the Investigation of Blast-related Traumatic Brain Injury. Front Neurol 4:16.

24. Schneiderman AI, Braver ER, Kang HK (2008) Understanding Sequelae of Injury Mechanisms and Mild Traumatic Brain Injury Incurred during the Conflicts in Iraq and Afghanistan: Persistent Postconcussive Symptoms and Posttraumatic Stress Disorder. Am J Epidemiol 167: 1446-52.

25. Benge JF, Pastorek NJ, Thornton GM (2009) Post-concussive Symptoms in OEF-OIF Veterans: Factor Sturcture and Impact of Post-traumatic Stress. Rehabil Psychol 54: 270-8.

26. Kennedy JE, Leal FO, Lewis JD, Cullen MA, Amador RR (2010) Posttraumatic stress symptoms in OIF/OEF service members with blast-related and non-blastrelated mild TBI. NeuroRehabilitation 26: 223-31.

27. Macera CA, Aralis HJ, Macgregor AJ, Rauh MJ, Galarneau MR (2012) Post-deployment symptom changes and traumatic brain injury and/or posttraumatic stress disorder in men. J Rehabil Res Dev 49: 1197-1208.

28. Whipple E, Greene J, Biester R, Robinson K, Nucifora P (2014) Using Multimodal Magnetic Resonance Imaging to Explore Biomarkers of Veterans with PostTraumatic Stress Disorder after Blast Injury. Arc Clin Neuropsychol 29: 595.

29. Garber BG, Rusu C, Zamorski MA (2014) Deployment-related mild traumatic brain injury, mental health problems, and post-concussive symptoms in Canadian armed forces personnel. BMC Psychiatry 14:325.

30. Hayes JP, Miller DR, Lafleche G, Salat DH, Verfaellie M (2015) The nature of white matter abnormalities in blast-related mild traumatic brain injury. Neuroimage: Clinical 8: 148-56.

31. Crowell TA, Kieffer KM, Siders CA, Vanderploeg RD (2002) Neuropsychological findings in combat-related post-traumatic stress disorder. Clin Neuropsychol 16: $310-21$.

32. Fear NT, Jones E, Groom M, Greenberg N, Hull L, et al. (2009) Symptoms of post-concussional syndrome are non-specifically related to mild traumatic brain injury in UK Armed Forces personnel on return from deployment in Iraq: an analysis of self-reported data. Psychological Medicine 39: $1379-87$.

33. Nelson NW, Hoelzle JB, McGuire KA, Ferrier-Auerbach AG, Charlesworth MJ, et al. (2010) Evaluation Context Impacts Neuropsychological Performance of OEF/OIF Veterans with Reported Combat-Related Concussion. Arch Clin Neuropsychol 25: 713-23.

34. Brenner LA, Ivins BJ, Schwab K, Warden D, Nelson LA, et al. (2010) Traumatic Brain Injury, Post-traumatic Stress Disorder, and Post-concussive Symptom Reporting Among Troops Returning From Iraq. J Head Trauma Rehabil 25: 307-12.

35. Luethcke CA, Bryan CJ, Morrow CE, Isler WC (2011) Comparison of concussion symptoms, cognitive performance, and psychological symptoms between acute blast-versus nonblast-induced mild traumatic brain injury. J Int Neuropsychol Soc 17: 36-45.

36. Belanger HG, Proctor-Weber Z, Kretzmer T, Kim M, French LM (2011) Symptom Complaints Following Reports of Blast Versus Non-Blast Mild TBI: Does Mechanism of Injury Matter? Clin Neuropsychol 25: 702-15.

37. Aupperle RL, Melrose AJ, Stein MB, Paulus MP (2012) Executive Function and PTSD: Disengaging from trauma. Neuropharmacology 62: 686-94.

38. Lange RT, Pancholi S, Brickell TA, Sakura S, Bhagwat A, et al. (2012) Neuropsychological outcome from blast-related versus non-blast related mild traumatic brain injury. J Int Neuropsychol Soc 18: 595-605.

39. Nelson NW, Hoelzle JB, Doane BM, McGuire KA, Ferrier-Auerbach AG, et al. (2012) Neuropsychological Outcomes of U.S. Veterans with Report of Remote Blast-Related Concussion and Current Psychopathology. J Int Neuropsychol Soc 18: 845-55.

40. Vasterling JJ, Brailey K, Proctor SP, Kane R, Heeren T, et al. (2012) Neuropsychological outcomes of mild traumatic brain injury, post-traumatic stress disorder and depression in Iraq-deployed US Army soldiers. Br J Psychiatry, 201:186-192.

41. Shandera-Ochsner AL, Berry DT, Harp JP, Edmundson M, Graue LO, et al. (2013) Neuropsychological Effects of Self-reported Deployment-related Mild TBI and Current PTSD in OIF/OEF Veterans. T Clin Neuropsychol, 27: 881-907.

42. Bolzenius JD, Roskos PT, Salminen LE, Paul RH, Bucholz RD (2015) Cognitive and Self-Reported Psychological Outcomes of Blast-Induced Mild Traumatic Brain Injury in Veterans: A Preliminary Study. Appl Neuropsychol Adult 22: 79-87.

43. Vasterling JJ1, Duke LM, Brailey K, Constans JI, Allain AN, et al. (2002) Attention, learning, and memory performances and intellectual resources in Vietnam veterans: PTSD and no disorder comparisons. Neuropsychology 16: 5-14.

44. Marx BP, Doron-Lamarca S, Proctor SP, Vasterling JJ (2009) The influence of pre-deployment neurocognitive functioning on post- deployment PTSD symptom outcomes among Iraq-deployed Army soldiers. J Int Neuropsychol Soc 15: 840-52.

45. Karr JE, Areshenkoff CN, Duggan EC, Garcia-Barrera MA (2014) Blast-Related Mild Traumatic Brain Injury: A Bayesian Random-Effects Meta - Analysis on the Cognitive Outcomes of Concussion among Military Personnel. Neuropsychol Rev 24: 428-44.

46. Troyanskaya M, Pastorek NJ, Scheibel RS, Petersen NJ, McCulloch K, et al. (2015) Combat Exposure, PTSD Symptoms, and Cognition Following Blast-Related Traumatic Brain Injury in OEF/OIF/OND Service Members and Veterans. Miltary Medicine 180: 285-9.

47. Bowen M, Littell C (1997) Discriminating adult normals, patients, and claimants with a pediatric test. Clini Neuropsychol 11: $433-5$.

48. Heaton RK, Grant I, Matthews CG (1991) Comprehensive Norms for an Expanded Halstead-Reitan Battery. Psychological Assessment Resources, Inc. Odessa, FL.

49. Thompson LL, Heaton RK, Matthews CG, Grant I (1987) Comparison of preferred and non-preferred hand performance on four neuropsychological motor tasks. Clin Neuropsychol 1: 324-34.

50. Llinas R, Ribary U, Contreras D, Pedroarena C (1998) The neuronal basis for consciousness. Philos Trans R Soc Lond B Biol Sci 353: $1841-9$.

51. Cavanna AE, Trimble MR (2006) The precuneus: a review of its functional anatomy and behavioural correlates. Brain 129: $564-83$.

52. Fromm-Auch D, Yeudall LT (1983) Normative data for the Halstead-Reitan neuropsychological tests. J Clin Neuropsychol 5: $221-238$.

53. Ghez C, Fahn S (1985) The cerebellum. In: Kandel ER, Schwartz JH, Principles of neuroscience ( $2^{\text {nd }}$ Edn) 502-21. 
54. Mac Donald CL, Johnson AM, Cooper D, Nelson EC, Werner NJ, et al. (2011) Detection of Blast-Related Traumatic Brain Injury in U.S. Military Personnel. N Engl J Med 364: 2091-100.

55. Mac Donald C, Johnson A, Cooper D, Malone T, Sorrell J, et al. (2013) Cerebellar White Matter Abnormalities following Primary Blast Injury in US Military Personnel. PLoS One 8: e55823.

56. Ramon-Cajal S (1995) Histology of the Nervous System of Man and Vertebrates. N Engl J Med 333: 1088.

57. Reitan RM (1959) Effects of Brain Damage on a Psychomotor Problem-Solving Task. Percept Mot Skills 9: 211-5.

58. Reitan RM, Wolfson O (1985) The Halstead-Reitan Neuropsychological Test Battery: Theory and clinical interpretation. Tucson, AZ: Neuropsychology Press.

59. Schmahmann, JD, Caplan D (2004) Cognition, emotion, and the cerebellum. Brain 129: 290-2.

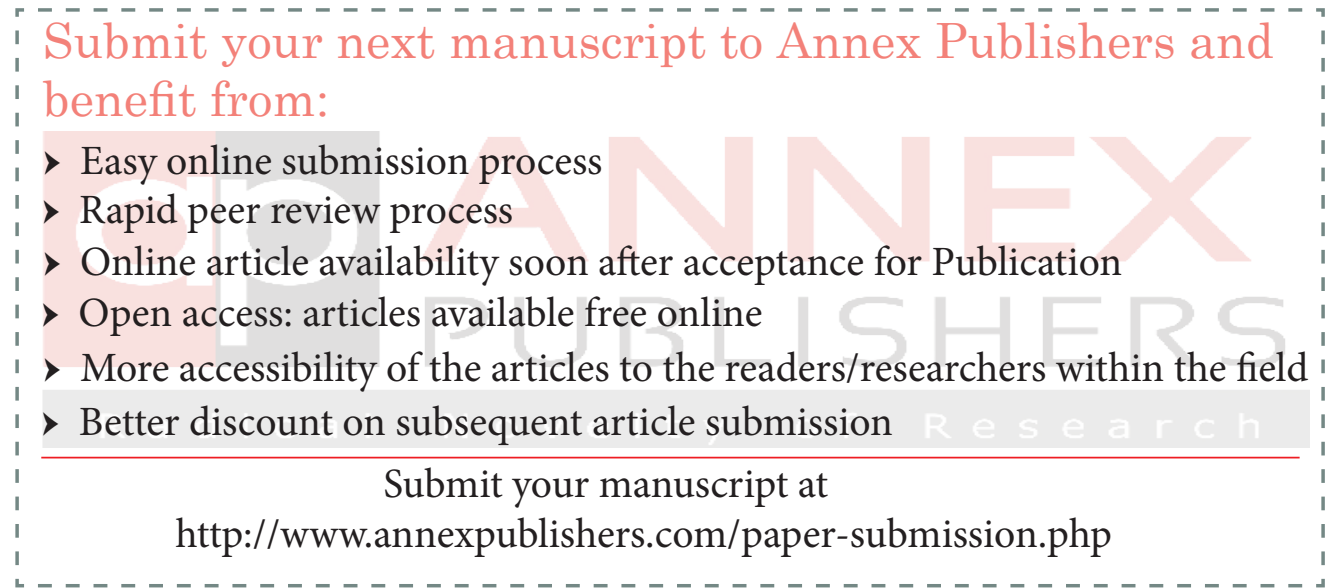

